\title{
IODINE DEFICIENCY AND PATHOLOGY OF THYROID GLAND AS A BACKGROUND STATE OF CANDIDIASIS OF MUCOSA OF THE UPPER PART OF DIGESTIVE TRACT
}

\author{
Inessa Kushnirenko \\ Department of Gastroduodenal Diseases, Dietology and Dietotherapy \\ SI «Institute of Gastroenterology NAMS of Ukraine» \\ 96 Slobozhansky ave., Dnipro, Ukraine, 49089 \\ inessa_mail@mail.ru
}

\begin{abstract}
There were examined 119 patients of gastroenterological profile, who, according to the results of microbiological examination of scraping from tongue and biopsy material of digestive tract and stomach, were divided into three groups: 1 group patients with oropharyngeal candidiasis and surface candidiasis of mucosa, 2 group - patients with invasion of Candida fungi in mucosa, 3 group - patients without oropharyngeal candidiasis and without growth of fungi in biopsy material. The status of iodine provision was studied in 78 persons. The results of research revealed that at structural changes of thyroid gland the dominating position in patients with candidiasis of mucosa occupies the nodular goiter- $29,31 \%$ and $36,36 \%$ for 1 and 2 group respectively, whereas in 3 group the frequency of nodular goiter was $11,76 \%$, at that hyperplasia and nodular goiter in patients with fungi invasion in mucosa was revealed 2,6 times more often comparing with patients without candidiasis $\left(\chi^{2}=4,01 ; p<0,05\right)$. In patients with oropharyngeal and surface candidiasis and invasive candidiasis of mucosa of the upper part of digestive tract the hard degree of iodine nutrition deficiency with thyroglobulin level higher than $40 \mathrm{ng} / \mathrm{ml}$ was revealed in more than half of cases. At that the frequency of hard iodine deficiency at fungi invasion in mucosa 4,2 times higher comparing with patients without candidiasis of mucosa $(\mathrm{F}=0,024 ; \mathrm{p}<0,05)$.

So, the concomitant comorbid state with pathology of thyroid gland and iodine deficiency is an aggravating factor in the course of candida infection that is necessary to be taken into account at clinical monitoring of patients with candidiasis of mucosa of the upper part of gastrointestinal tract.
\end{abstract}

Keywords: candidiasis, mucosa, upper part of digestive tract, pathology of thyroid gland, immunity, iodine deficiency.

\section{Introduction}

Candidiasis of the mucosa of the upper part of digestive tract is a disease, which development is connected with excessive growth of Candida fungi that are opportunistic flora for human. The condition of that is, first of all, the lowering of macroorganism resistance [1-3]. The features of immune protection of macroorganism determine the further course of infection - colonization or invasion of epithelium $[4,5]$. According to several researches, T-lymphocytic link is a dominating one at the development of immune response at oral and gastrointestinal candidiasis and interaction between Candida albicans and epithelial cells induces the defense of mucosal barrier by activation of immune cells and production of cytokines and chemokines, among which are IL-1 $\alpha$, IL-1 $\beta$, IL-6, IL-8, [6-9]. Except the features of immune protection and hard immunodificient disease such as HIV, among risk factors of Candidaspp growth in the upper part of digestive tract are indicated the age of patient, use of removable dental prostheses, deficiency of iron, folates, vitamin B12 [10-12]. And among endocrine diseases are indicated diabetes and autoimmune multiple endocrine syndrome [2]. The recent studies demonstrated the role of pathology of thyroid gland in regulation of immune response by mediated influence on dendritic cells and their ability to cause proliferation of T-cells and induce the cytokines production [13, 14]. There are also data about deficiency of iodine nutrition as an important factor of immunodeficiency development and the several ones demonstrate the possibility of iodine preparations to strengthen T-cellular link of immune protection $[15,16]$. A lot of attention is paid to the problem of iodine deficiency because of its spread; thus, according to WHO data, 740 millions of people in the world suffer from iodine deficiency that is $13,0 \%$ of human population [17]. According to the data of Ukrainian researchers, 
Dnipro region is referred to the ones with weak and moderate iodine deficiency [18]. The level of iodine nutrition is determined by the level of thyroblobulin that is reliable and sensitive marker of iodine nutrition status of macroorganism [19-21]. But the connection between the status of thyroid gland, iodine provision and indices of adaptive and innate immunity in the aspect of interaction at candidiasis of mucosa of the upper part of digestive tract stays undetermined.

\section{Aim of research}

To define the structural state of thyroid gland and the level of iodine nutrition in patients with candidiasis of mucosa of the upper part of digestive tract and to assess their influence of indices of innate and adaptive immunity in these patients.

\section{Materials and Methods}

There was carried out an examination of 119 patients, who were on treatment in the department of diseases of stomach and duodenum. According to the results of microbiological examination, patients were divided into three groups: 1 group - 58 patients with IV degree of massivity of Candida fungi seeding that is oropharyngeal candidiasis (OPC) and surface growth of fungi in material from the mucosa of digestive tract and/or stomach; 2 group -44 patients with invasive growth of Candida fungi in mucosa of digestive tract and/or stomach; 3 group - 17 patients without OPC and without fungi growth in biopsy material. The criterion of exclusion from the research was the positive HIV-status. Mean age of patients was $(47,00 \pm 12,22),(50,62 \pm 15,24)$ and $(46,33 \pm 13,34)$ years in 1,2 and 3 groups, respectively. The women-men ratio was 2,2:1,5:1,4 in all three groups, respectively. Patients underwent ultrasound examination of thyroid gland. Subpopulational composition of lymphocytes was determined with the help of monoclonal antibodies made by "Sorbent TM" to the CD3+, CD4+, CD8+, CD16+, CD22+ markers with the help of lymphocytotoxic test (NIH standard method, USA). The content of lactoferrin and secretory immunoglobulin A (IgA) in gastric juice and saliva was determined using standard sets made by CC "Vector-Brest" (Russia). The study of thyroglobulin and thyrotropic hormone level in blood serum was carried out by the method of solid phase immune-enzyme assay in 41 patients of 1 group, 31 ones of 2 group and in 6 persons of 3 group. The degree of severity of iodine deficiency was determined according to WHO recommendations, 1994. Statistical analysis was carried out using Fisher (F) criterion, Student's t-criterion. Correlative analysis using Pirson's (r) correlation coefficient for parametric values and Spearman's $(\rho)$ one for nonparametric values was carried out in group of 78 patients with determined status of iodine nutrition and in group of 48 patients, who underwent the analysis of efficiency of treatment arrangements as to candidiasis of mucosa of the upper part of digestive tract.

\section{Results of research}

The results of study of the structural features of thyroid gland by ultrasound method are presented on the Fig. 1.

As we can see, the frequency of normal structure of thyroid gland was only near the third of examined of 2 group and in patients of 1 and 3 group - near 40 percents of examined. The structural changes such as nodular goiter occupied dominating position in patients of 1 and 2 group and were registered in $29,31 \%(\mathrm{n}=17)$ and $36,36 \%(\mathrm{n}=16)$, respectively, whereas in 3 group the frequency of nodular goiter was $11,76 \%(\mathrm{n}=2)$. In whole hyperplasia and nodular goiter in patients with fungi invasion in mucosa was revealed 2,6 times more often comparing with patients without candidiasis $\left(\chi^{2}=4,01 ; \mathrm{p}<0,05\right)$.

Correlative analysis demonstrated the connection of thyroid gland pathology with series of factors that plays role in development and course of candida infection. Thus, the nodular goiter was associated with increase of frequency of revelation of Candida fungi on the mucosa of stomach body $(\rho=0,216 ; p=0,030)$. Hyperplasia of thyroid gland has a negative impact on formation of cellular link of adaptive and innate immunity that is manifested by the negative correlations with $\mathrm{CD} 8+(\mathrm{r}=-0,242 ; \mathrm{p}=0,021)$ and $\mathrm{CD} 16+(\mathrm{r}=-0,231 ; \mathrm{p}=0,027)$ levels. 


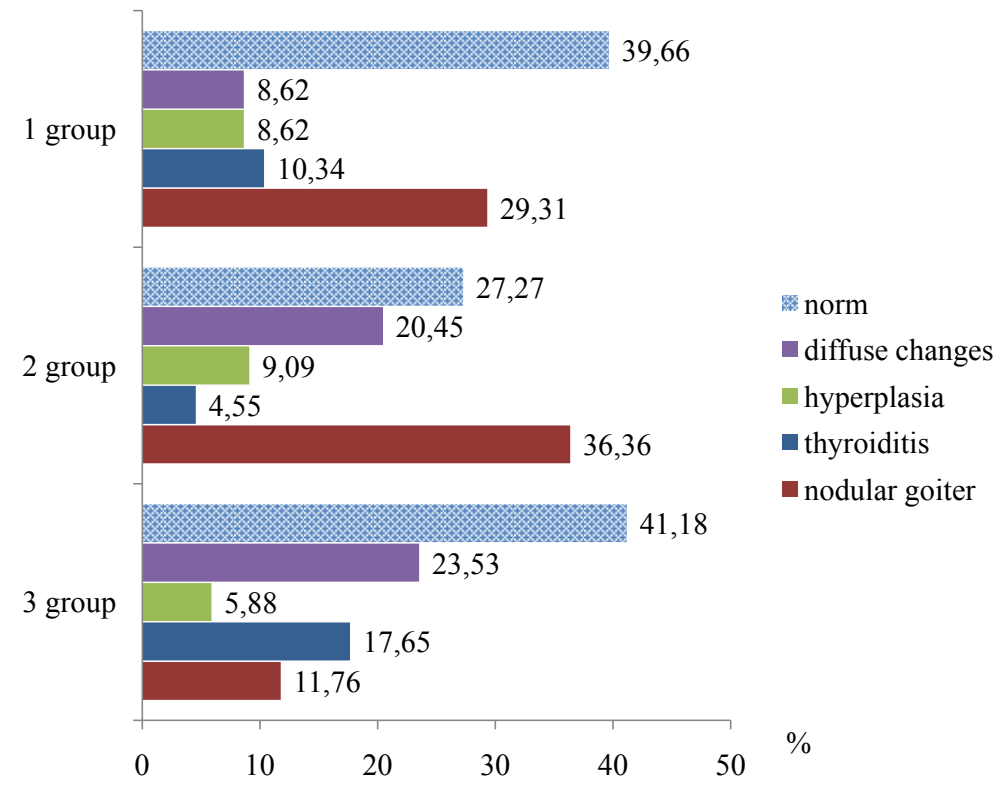

Fig. 1. Characteristic of structural state of thyroid gland in examined patients according to the results of ultrasound examination

The mean level of thyrotropic hormone in groups was $(2,02 \pm 0,17) \mathrm{mIU} / \mathrm{ml}$, in 2 $(1,93 \pm 0,15) \mathrm{mIU} / \mathrm{ml}$ and in $3-(1,56 \pm 0,33) \mathrm{mIU} / \mathrm{ml}$. The increase of its level was revealed in 2 patients of 1 group $-4,44 \%$, in 1 person of 2 group $(2,56 \%)$.

Calculation of the mean value of thyroglobulin concentration in blood serum demonstrated its significant increase in patients of 1 and 2 groups comparing with 3 one (Fig. 2).

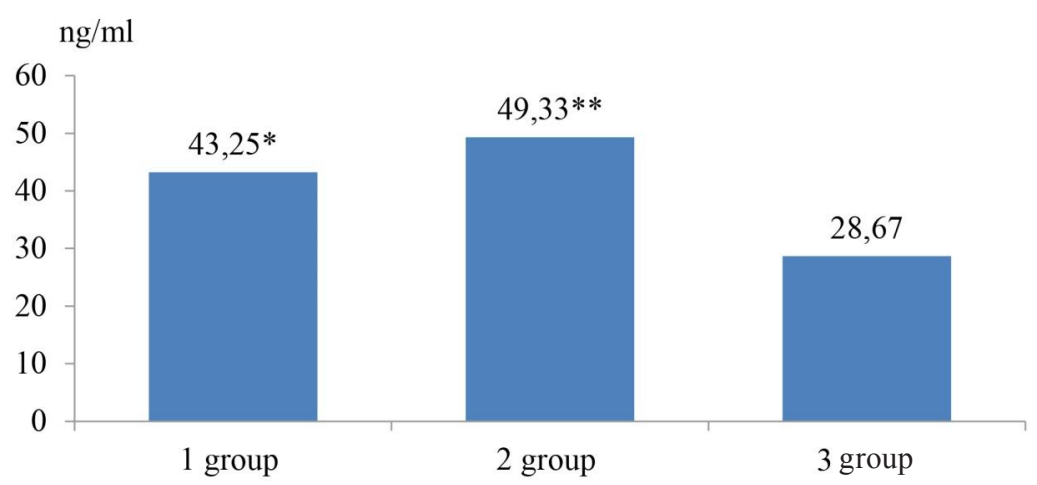

Fig. 2. Thyroglobulin level in groups $(\mathrm{M} \pm \mathrm{m}),(\mathrm{ng} / \mathrm{ml}):{ }^{*}-\mathrm{p}<0,01-$ reliability of the difference comparing with 3 group by Student's t-criterion; $*^{*}-\mathrm{p}<0,001$ - reliability of the difference comparing with 3 group by Student's t-criterion

As we can see, thyroglobulin level in patients of 1 and 2 group was within severe iodine deficiency, whereas in patients of 3 one it corresponded to the limits of the middle degree of intensity. Characteristic of the status of iodine nutrition by the degree of deficiency intensity is presented on the Fig. $\mathbf{3}$.

The analysis testifies to the rather high frequency and degree of severity of revealed iodine deficiency in examined patients of gastroenterological profile in whole, because the normal thyroglobulin level was registered only in one patient. Analysis of groups demonstrated, that if in the group of patients without candidasis of mucosa dominated the middle level of severity of iodine deficiency with thyroglobulin level from 20,0 to $40,0 \mathrm{ng} / \mathrm{ml}$, in patients with OPC and surface candidiasis and invasive candidiasis of mucosa of the upper part of digestive tract in more than half of cases was revealed the hard degree of iodine nutrition deficiency with thyroglobulin level more than $40 \mathrm{ng} / \mathrm{ml}$. At that such raise acquired reliability at comparison of 2 and 3 group - the frequency of hard iodine 
deficiency at fungi invasion in mucosa is 4,2 times higher comparing with patients without candidiasis of mucosa $(\mathrm{F}=0,024 ; \mathrm{p}<0,05)$.

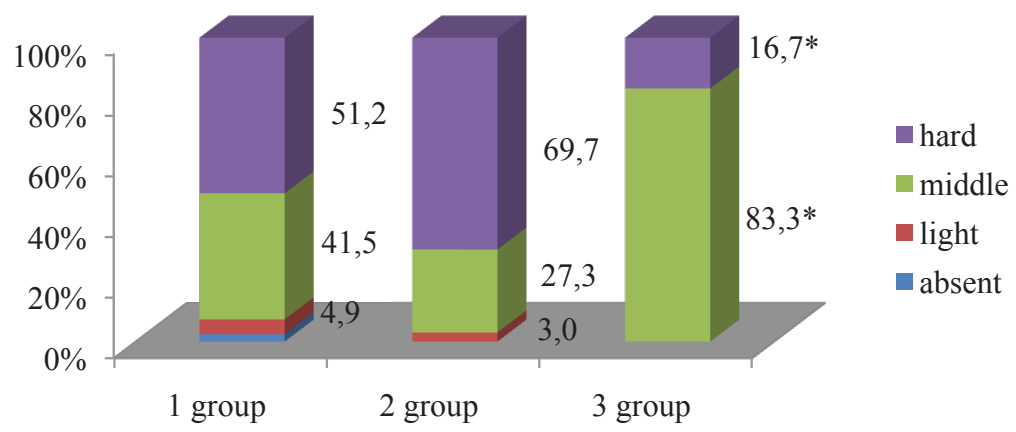

Fig. 3. Characteristic of iodine deficiency by thyroglobulin level in examined patients in groups: $*-p<0,05$ - reliability of the difference comparing with 2 group by F-criterion

The raise of thyroglobulin was associated with increase of frequency of revelation of Candida albicans growth on surface of mucosa of digestive tract $(r=0,252 ; p=0,033)$, and increase of iodine deficiency degree - with more frequency of revelation of fungi invasion in mucosa of the upper part of digestive tract $(\rho=0,394 ; p=0,011)$. The raise of thyroglobulin was also associated with the raise of $\mathrm{CD} 22+(\mathrm{r}=0,337 ; \mathrm{p}=0,007)$ and had a negative impact on balance of $\mathrm{T}$ - and B-lymphocytes that is manifested by the negative correlation with CD3+/CD22+ index $(r=-0,256 ; p=0,044)$. In its turn the increased CD22+ content before treatment is connected with the negative effect from therapy $(r=0,618 ; p=0,006)$.

There is revealed a correlation between thyroglobulin level and iodine deficiency degree with series of parameters that determine a possibility of receiving the positive response to the therapy of patients with candidiasis. Thus, the increase of thyroglobulin level was associated with decrease of secretory IgA in saliva of examined persons before treatment $(r=-0,921 ; p=0,026)$. And the low level of $\operatorname{IgA}$ in oral cavity of examined before treatment causes the preservation of fungi growth on surface and in biopsy material of stomach body after treatment $(r=-0,828 ; p=0,042)$ and $(r=-0,828 ; p=0,042)$, respectively, and Candida albicans surface growth in the antral part of stomach after treatment $(\mathrm{r}=-0,828 ; \mathrm{p}=0,042)$. At the same time the increase of severity degree of iodine deficiency causes lowering of the level of lactoferrin, humoral component of iinate immunity, in the gastric juice $(r=-0,433 ; p=0,044)$ before treatment that lowers a possibility of resistance to infection already at the stage of primary contact with infectious agent.

The correlation between the hyperplasia of thyroid gland and nodular goiter and the absence of satisfactory response to treatment in whole is important $(\rho=0,479 ; p=0,039)$.

\section{Discussion of results}

So, according to the results of studies, it was determined, that in patients with candidiasis of mucosa of the upper tract in near the third of cases is revealed hyperplasia of thyroid gland and nodular goiter that is more often comparing with patients without candidiasis of mucosa. These changes take place on the background of intense iodine deficiency, determined by the thyroglobulin level. Moreover in patients with candidiasis of mucosa the hard degree of iodine deficiency, that is not typical for Dnipro region of Ukraine, was registered three times more often in the group with oropharyngeal and surface candidiasis and 4,2 times more often in group with Candida albicans invasion in mucosa comparing with patients without candidiasis. The thyrotropic hormone level in examined patients was registered within norm and only in several patients exceeded the threshold values $(n=3)$.

It is known, that the sufficient level of lactoferrin in saliva as essential component of system of innate immunity is necessary for effective resistance to infection already at the stage of entrance to human organism. The series of researches demonstrated the important protective role of this protein not only in prevention of adhesion of candida to epithelial cells and in realization of effective mod- 
ulation of both innate cellular immunity at the expanse of activation of macrophages, natural killer and adaptive one - at the expanse of ability to increase the level of CD3+ T-cells and CD4+ T-cells and inhibit activation of CD22+ B-lymphocytes that is favorable for the course of candida infection $[23,24]$. Our studies demonstrated the negative correlation between iodine deficiency degree and lactoferrin level in gastric juice $(p<0,05)$, that is a proof of influence of this state on functioning of congenital immunity components in the liquid mediums of upper part of digestive tract at candidiasis of mucosa. The negative correlation was also revealed between the thyroglobulin level, that was significantly higher in patients with candidiasis of mucosa comparing with ones without it, and secretory IgA in saliva of examined patients $(\mathrm{p}<0,05)$. The value of secretory IgA in protection from fungal infection is in ability to inhibit the Candida albicans adhesion to epithelial cells that plays an important role in the local control of fungi growth in the oral cavity [25]. But according to the data of other researchers, in experimental model of candida infection was not received the raise of secretory IgA level after oral immunization against Candida albicans that put in their minds an idea about the presence of protectoral effect of humoral link of immune defense as to oropharyngeal candidasis [26]. But from our point of view, it can be connected with mediated mechanisms of regulation of its synthesis by the dendritic cells, among which the differentiation of B-lymphocytes that are responsible for its synthesis and the ability of epithelial cells to the synthesis of glycoprotein that provides stability of its structure. Interconnection between thyreoglobulin and secretory $\operatorname{Ig} \mathrm{A}$ levels testifies to the influence of iodine nutrition deficiency on the functional activity of humoral link of immune protection at candidiasis of mucosa of the upper part of gastrointestinal tract. The direct correlation between thyroglobulin level and synthesis of B-lymphocytes $(p<0,01)$, that in its turn is associated with negative response to treatment $(p<0,01)$, allow think about the necessity of the further study of formation of human immune response at the contact with Candida albicans infection at iodine deficiency and the necessity of its timely revelation and correction for receiving the positive therapeutic effect and improvement of medical care.

\section{Conclusions}

1. Concomitant comorbid state with pathology of thyroid gland and iodine deficiency is an aggravating factor in the course of candida infection that is necessary to be taken into account at clinical monitoring of patients with candidiasis of mucosa of the upper part of gastrointestinal tract.

2 . The structural changes of thyroid gland such as hyperplasia and nodular goiter are associated with lowering of indices of innate (CD16+) and adaptive (CD8+) immunity.

3. The raise of thyroglobulin level and iodine nutrition deficiency are connected with indices of innate (lactoferrin) and adaptive (CD22+, secretory IgA) immunity in patients with candidiasis of mucosa of the upper part of digestive tract that influences the efficiency of treatment of this group of patients.

4. The further researches are necessary to study the mechanisms of influence of iodine deficiency on the system of immune protection of macroorganism at candidiasis of mucosa of the upper part of digestive tract.

\section{References}

[1] Krishnan, Pa. (2012). Fungal infections of the oral mucosa. Indian Journal of Dental Research, 23 (5), 650-659. doi: 10.4103/0970-9290.107384

[2] Patil, S., Rao, R. S., Majumdar, B., Anil, S. (2015). Clinical Appearance of Oral Candida Infection and Therapeutic Strategies. Frontiers in Microbiology, 6. doi: 10.3389/fmicb.2015.01391

[3] Ohshima, T., Kojima, Y., Seneviratne, C. J., \& Maeda, N. (2016). Therapeutic Application of Synbiotics, a Fusion of Probiotics and Prebiotics, and Biogenics as a New Concept for Oral Candida Infections: A Mini Review. Frontiers in Microbiology, 7. doi: 10.3389/fmicb.2016.00010

[4] Gow, N. A., Hube, B. (2012). Importance of the Candida albicans cell wall during commensalism and infection. Current Opinion in Microbiology, 15 (4), 406-412. doi: 10.1016/j.mib.2012.04.005

[5] Neil, A. R., van de Veerdonk, F. L., Brown, A. J. P., Netea, M. G. (2012). Candida albicans morphogenesis and host defence: discriminating invasion from colonization. Nature Reviews Microbiology, 10 (2): 112-122. doi: $10.1038 /$ nrmicro2711 
[6] Hebecker, B., Naglik, J. R., Hube, B., Jacobsen, I. D. (2014). Pathogenicity mechanisms and host response during oral Candida albicans infections . Expert Review of Anti-Infective Therapy, 12 (7), 867-879. doi: 10.1586/14787210.2014.916210

[7] Salvatori, O., Puri, S., Tati, S., Edgerton, M. (2016). Innate Immunity and Saliva in Candida albicans-mediated Oral Diseases. Journal of Dental Research, 95 (4), 365-371. doi: 10.1177/0022034515625222

[8] Ashman, R. B., Farah, C. S., Wanasaengsakul, S., Hu, Y., Pang, G., Clancy, R. L. (2004). Innate versus adaptive immunity in Candida albicans infection. Immunology and Cell Biology, 82 (2), 196-204. doi: 10.1046/j.0818-9641.2004.01217.x

[9] Naglik, J. R., Fidel, P. L., Odds, F. C. (2008). Animal models of mucosal Candida infection. FEMS Microbiology Letters, 283 (2), 129-139. doi: 10.1111/j.1574-6968.2008.01160.x

[10] Ellepola, A. N. B., Samaranayake, L. P. (2000). Oral Candidal Infections and Antimycotics. Critical Reviews in Oral Biology \& Medicine, 11 (2), 172-198. doi: 10.1177/10454411000110020301

[11] Yakoob, J. (2003). Candida esophagitis: Risk factors in non-HIV population in Pakistan. World Journal of Gastroenterology, 9 (10), 2328-2331. doi: 10.3748/wjg.v9.i10.2328

[12] Bianchi, C. M. P. de C., Bianchi, H. A., Tadano, T., Paula, C. R. de, Hoffmann-Santos, H. D., Leite Jr, D. P., Hahn, R. C. (2016). Factors related to oral candidiasis in elderly users and non-users of removable dental prostheses. Revista do Instituto de Medicina Tropical de São Paulo, 58. doi: 10.1590/s16789946201658017

[13] Hodkinson, C. F., Simpson, E. E. A., Beattie, J. H., O’Connor, J. M., Campbell, D. J., Strain, J. J., Wallace, J. M. W. (2009). Preliminary evidence of immune function modulation by thyroid hormones in healthy men and women aged 55-70 years. Journal of Endocrinology, 202 (1), 55-63. doi: 10.1677/joe-08-0488

[14] De Vito, P., Incerpi, S., Pedersen, J. Z., Luly, P., Davis, F. B., Davis, P. J. (2011). Thyroid Hormones as Modulators of Immune Activities at the Cellular Level. Thyroid, 21 (8), 879-890. doi: 10.1089/thy.2010.0429

[15] Venturi, S., Venturi, M. (2009). Iodine in Evolution of Salivary Glands and in Oral Health. Nutrition and Health, 20 (2), 119-134. doi: 10.1177/026010600902000204

[16] Miller D. W. (2006). Extrathyroidal bemefits of Iodine. Journal of American Physicians and Surgeons, 11 (4), 106-110.

[17] Iodine and inorganic iodides: human health aspects. (Concise international chemical assessment document; 72) (2009). WHO Library Cataloguing-in-Publication Data, World Health Organization, 54.

[18] Bondar', T. N. (2008). Subklinicheskie narushenija funkcii shhitovidnoj zhelezy. Annals of Mechnikov Institute, 4, 9-13.

[19] Zimmermann, M. B. (2009). Iodine Deficiency. Endocrine Reviews, 30 (4), 376-408. doi: 10.1210/ er.2009-0011

[20] Vejbjerg, P., Knudsen, N., Perrild, H., Laurberg, P., Carle, A., Pedersen, I. B. et. al. (2009). Thyroglobulin as a marker of iodine nutrition status in the general population. European Journal of Endocrinology, 161 (3), 475-481. doi: 10.1530/eje-09-0262

[21] Zimmermann, M. B., Aeberli, I., Andersson, M., Assey, V., Yorg, J. A. J., Jooste, P. et. al. (2013). Thyroglobulin Is a Sensitive Measure of Both Deficient and Excess Iodine Intakes in Children and Indicates No Adverse Effects on Thyroid Function in the UIC Range of 100-299 $\mu \mathrm{g} / \mathrm{L}$ : A UNICEF/ICCIDD Study Group Report. The Journal of Clinical Endocrinology \& Metabolism, 98 (3), 1271-1280. doi: 10.1210/jc.2012-3952

[22] Legrand, D., Mazurier, J. (2010). A critical review of the roles of host lactoferrin in immunity. BioMetals, 23 (3), 365-376. doi: 10.1007/s10534-010-9297-1

[23] Garcia-Montoya, I. A., Cendón, T. S., Arevalo-Gallegos, S., Rascon-Cruz, Q. (2012). Lactoferrin a multiple bioactive protein: An overview. Biochimica et Biophysica Acta (BBA) - General Subjects, 1820 (3), 226-236. doi: 10.1016/j.bbagen.2011.06.018

[24] Ueta, E., Tanida, T., Doi, S., Osaki, T. (2000). Regulation of Candida albicans growth and adhesion by saliva. Journal of Laboratory and Clinical Medicine, 136 (1), 66-73. doi: 10.1067/mlc.2000.107304

[25] Farah, C. S., Ashman, R. B. (2005). Active and passive immunization against oral Candida albicans infection in a murine model. Oral Microbiology and Immunology, 20 (6), 376-381. doi: 10.1111/j.1399302x.2005.00240.x 